\title{
Kryo-Elektronenmikroskopie
}

\section{Protein-Cross-Linking zur Aufklärung von komplexen Strukturen}

\author{
OLEXANDR DYBKOV ${ }^{1}$, ALEXANDRA STÜTZER ${ }^{2}$, KARL BERTRAM ${ }^{3}$, BERTHOLD \\ KASTNER $^{1}$, HOLGER STARK ${ }^{3}$, REINHARD LÜHRMANN ${ }^{1}$, HENNING URLAUB ${ }^{2,} 4$ \\ ${ }^{1}$ ABTEILUNG ZELLULÄRE BIOCHEMIE, MAX-PLANCK-INSTITUT FÜR \\ BIOPHYSIKALISCHE CHEMIE, GÖTTINGEN \\ ${ }^{2}$ BIOANALYTISCHE MASSENSPEKTROMETRIE, MAX-PLANCK-INSTITUT FÜR \\ BIOPHYSIKALISCHE CHEMIE, GÖTTINGEN \\ ${ }^{3}$ ABTEILUNG STRUKTURELLE DYNAMIK, MAX-PLANCK-INSTITUT FÜR \\ BIOPHYSIKALISCHE CHEMIE, GÖTTINGEN \\ ${ }^{4}$ BIOANALYTIK, INSTITUT FÜR KLINISCHE CHEMIE, UNIVERSITÄTSMEDIZIN \\ GÖTTINGEN
}

\section{Cryo-electron microscopy (cryo-EM) can solve structures of highly dynam- ic macromolecular complexes. To characterize less well defined regions in cryo-EM images, cross-linking coupled with mass spectrometry (CX-MS) provides valuable information on the arrangement of domains and amino acids. CX-MS involves covalent linkage of protein residues close to each other and identifying these connections by mass spectrometry. Here, we summarise the advances of CX-MS and its integration with cryo-EM for structural reconstruction.}

DOI: $10.1007 / \mathrm{s} 12268-018-0909-6$ (C) Die Autoren 2018

Proteine sind dynamische Moleküle, die oft mit Liganden und anderen Proteinen funktionelle makromolekulare Komplexe in der Zelle bilden. Die Bestimmung der 3D-Struktur solcher Komplexe stellt eine Herausforderung dar. Klassische strukturgebende Verfahren, wie Röntgenkristallographie und Magnetresonanzspektroskopie, sind aufgrund der molekularen Größe, der Flexibilität und der Heterogenität der meisten makromolekularen Proteinkomplexe limitiert.

Derzeit etabliert sich die Kryo-Elektronenmikroskopie (Kryo-EM) zur Strukturaufklärung von makromolekularen Komplexen. Die Kryo-EM ermöglicht die 3DStrukturaufklärung aus 2D-Bildprojektionen, wobei die Struktur der zu untersuchenden Spezies durch die Einbettung in amorphem Eis in einem möglichst nativen Zustand erhalten bleibt. Durch technische Verbesserungen an den Mikroskopen sowie die Entwicklung geeigneter Software wer- den derzeit beindruckende Strukturen von teilweise unter 3 Ångström ( $)$ Auflösung erreicht [1-4]. Beispiele solcher Strukturen sind unter anderem Ribosomen, Proteasomen, an der Transkription beteiligte Komplexe, Spleißosomen, Kernporen und mitochondriale, membranständige Importkomplexe [5].

Dennoch besitzt nur ein sehr kleiner Teil solcher Strukturen eine Auflösung von $3 \AA$ oder besser. Mehr als 90 Prozent der veröffentlichten Strukturen besitzen eine Auflösung von 3 bis $5 \AA$ oder schlechter (https://pdbj.org/emnavi/stat.php). Die Gründe hierfür sind Bewegungsfreiheitsgrade der Proteinkomponenten innerhalb des makromolekularen Komplexes sowie innerhalb der einzelnen Proteine. Ferner spielt die Probenqualität, die Qualität der 2D-Bilder und die Statistik des 3D-Bildprozesses eine Rolle. Da bei Strukturen mit einer Auflösung von schlechter als $4 \AA$, nicht die Seitenketten der
Aminosäuren sichtbar sind, ist die Lokalisation und Anordnung von Proteinen in KyroEM-Strukturen bei entsprechend geringerer Auflösung erschwert.

Hier unterstützt die chemische Proteinquervernetzung (Cross-Linking) in Verbindung mit Massenspektrometrie (MS) die 3DKryo-EM. Cross-Linking in Verbindung mit MS (CX-MS) ermöglicht es, Aussagen über die interagierenden Proteindomänen in makromolekularen Komplexen zu erhalten, nachdem diese chemisch quervernetzt und die quervernetzten Proteinregionen mittels MS identifiziert und sequenziert wurden [68]. CX-MS ist in diesem Sinne keine eigenständige Methode zur Strukturaufklärung, aber sie trägt in hohem Maße zur Interpretation von Strukturen bei. Dies gilt insbesondere, wenn (1) es keine strukturellen Informationen zur Anordnung von Proteinen in Komplexen gibt, (2) Proteine sehr dynamische Domänen enthalten, die nicht durch Kristallographie oder Kernspinresonanz(NMR)Spektroskopie erfasst werden können und auch in Kryo-EM keine Zuordnung zu Proteinstrukturen gestatten, (3) Komplexe nur mit einem Teil ihrer Proteine strukturell bestimmt werden können, während andere spezifische Proteinkomponenten nicht in der Struktur erfasst werden können, oder (4) zur Validierung von Strukturvorhersagen von Strukturmodellen von Proteinen. Zudem ermöglicht CX-MS die Identifizierung von verschiedenen Proteinkonformationen einzelner Proteine in einem Komplex, wenn sich durch Interaktionen mit anderen Proteinen die Quervernetzungsstellen ändern.

\section{Protein-Cross-Linking}

Für das Cross-Linking werden meist Reagenzien verwendet, die als reaktive Gruppen N-Hydroxysuccinimid(NHS)-Ester besitzen und mit primären Aminogruppen von Lysinen wie auch mit dem N-Terminus des Proteins reagieren (Abb. 1). NHS-Ester sind relativ stabil in wässriger Lösung und bei neutralem pH-Wert reaktiv. Ferner befinden sich Lysinreste bevorzugt auf den Oberflächen von 
Proteinen, was sie zum idealen Angriffspunkt für die Reagenzien macht. Die NHS-EsterCross-Linker besitzen einen Spacer zwischen den reaktiven Gruppen von variabler Länge. Das am meisten verwendete Reagens Disuccinimidylsuberat (DSS) vernetzt Lysine, die ca. $30 \AA$ A voneinander entfernt sind, wobei der Abstand der jeweiligen $\mathrm{C}_{\alpha}$-Atome der Lysine gemeint ist. Nach chemischer Quervernetzung werden die Proteine enzymatisch mittels einer Endoproteinase (meist Trypsin) verdaut und die quervernetzten Peptide samt Cross-Linker im Massenspektrometer sequenziert. Aus der Peptidsequenz ergeben sich die quervernetzten Aminosäuren (z. B. Lysine) und die quervernetzten Proteine. Die Datenbanksuche nach der Sequenzierung der quervernetzten Peptide im Massenspektrometer stellt, anders als bei der etablierten MS-basierten Proteomik, eine enorme Herausforderung dar. Es bedarf spezieller Software (Tab. 1), die nicht nur eine einzelne Sequenz, sondern beide quervernetzten Peptidsequenzen (plus der molekularen Massen des verwendeten Cross-Linkers) aus den Kombinationen aller infrage kommenden Peptidsequenzen aller im Komplex vorliegenden Proteine ermittelt und mit den Sequenzierdaten abgleicht.

Um die Datenbanksuche zu vereinfachen, wurden spaltbare Cross-Linker entwickelt (Abb. 1, [9-11]). Solche Cross-Linker wie Disuccinimidylsulfoxid (DSSO) sind in der Gasphase des Massenspektrometers spaltbar. Damit wird wie bei der klassischen Peptidsequenzierung das quervernetzte Peptidpaar ausgewählt, aber zunächst nur der Cross-Linker gespalten, während die Peptide intakt bleiben. Im zweiten Schritt werden die beiden Peptide ausgewählt und anschließend unabhängig voneinander sequenziert. Das hat den Vorteil, dass beide Sequenzen mit einer normalen Datenbanksuche (unter Berücksichtigung des Restes des Cross-Linkers) identifiziert werden können.

Ähnlich stabile Cross-Linker wie NHSEster-basierte Reagenzien beinhalten Maleimidgruppen und reagieren bevorzugt mit Cystein. Andere Cross-Linker reagieren mit den Carboxylgruppen der Aminosäuren, aber ihre Verwendung ist aufgrund der meist sauren Reaktionsbedingungen limitiert. Des Weiteren existieren UV-reaktive ProteinCross-Linker. Diese reagieren mit jeder Aminosäure, was die massenspektrometrische Datenauswertung erschwert, da die reaktive Aminosäure nicht a priori definiert werden kann.

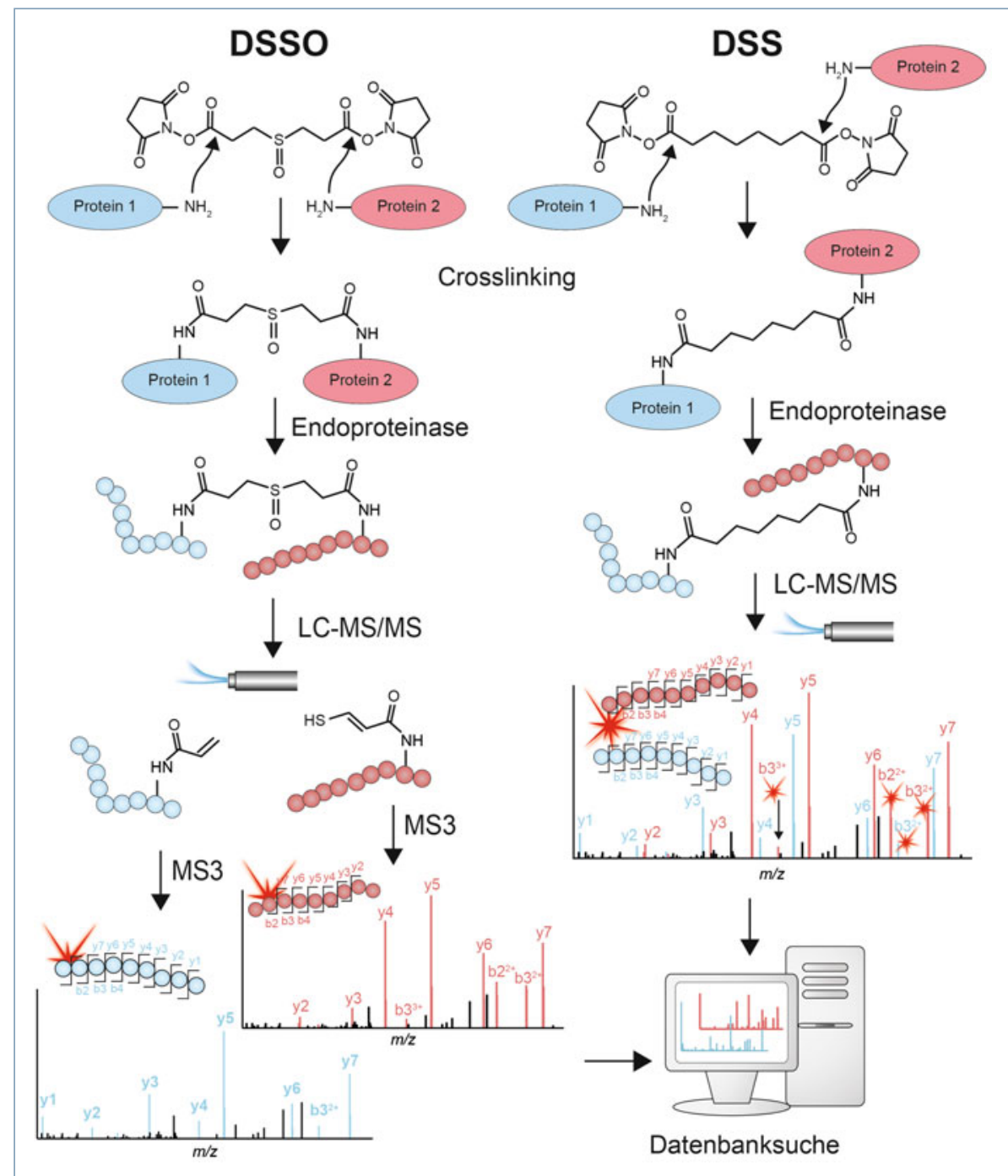

$\Delta$ Abb. 1: Schematische Darstellung der chemischen Quervernetzungsreaktion von Proteinen mit Disuccinimidylsulfoxid (DSSO) und Disuccinimidylsuberat (DSS) und des anschließenden Verdaus mit Endoproteinase zur massenspektrometrischen Analyse der quervernetzten Peptide. In Proteinen reagieren die Aminogruppen von Lysinen mit der N-Hydroxysuccinimid(NHS)-Ester-Gruppe des Quervernetzungsreagens, wobei die NHS-Gruppe abgespalten wird und eine stabile Amidbindung entsteht. Sind zwei Proteine in räumlicher Nähe zueinander, kann zwischen den beiden Proteinen eine kovalente Bindung ausgebildet werden (Cross-Link). Nach enzymatischem Verdau werden die quervernetzen Peptide im Massenspektrometer sequenziert (LC-MS/MS) und das entsprechende Protein durch Datenbanksuche identifiziert. Dadurch lässt sich ermitteln, welche Proteine und Proteinregionen in Nachbarschaft zueinander liegen. Bei nicht spaltbaren Cross-Linkern werden zwei Peptide auf einmal im MS sequenziert, während bei spaltbaren Cross-Linkern dieser zunächst im MS gespalten wird und die ursprünglich quervernetzten Peptide separat voneinander sequenziert werden (MS3).

CX-MS von Proteinkomplexen erfolgt wie in Abbildung 2 dargestellt. Isolierte, makromolekulare Proteinkomplexe werden mit Cross-Linker-Reagenzien inkubiert, sodass Proteine, die in Nachbarschaft zueinander liegen, quervernetzt werden (Interlinks). Darüber hinaus werden Proteine auch in sich vernetzt (Intralinks). Quervernetzte Komplexe werden mit Trypsin verdaut und die quervernetzten Peptidpaare angereichert, z. B. durch eine Gelfiltration. Angereicherte Fraktionen werden im Massenspektrometer analysiert und die quervernetzten Peptidpaare sequenziert. Durch die Sequenzierung werden die quervernetzten Proteine mit ihrer jeweils quervernetzten Aminosäure identifiziert. Der Einsatz von spezieller Software ermöglicht die Visualisierung der identifizierten Quervernetzungsstellen auf Proteinebene und in 3DStrukturmodellen der Proteine (Tab. 1). 
Tab. 1: Verschiedene Softwares mit den entsprechenden URLs zur massenspektrometrischen Identifizierung und Darstellung von Protein-Protein-Crosslinks und zur Modellierung. Die Literaturangaben zu diesen Softwares befinden sich auf den entsprechenden Internetseiten.

Software zur Identifizierung von Crosslinks

ECL 2.0
Kojak
Mass Spec Studio
MaxQuant
MeroX
pLink1 / pLink2
Protein Prospector
SIM-XL
StavroX
xComb
Xilmass

XlinkX/Proteome Discoverer 2.2

xQuest/xProphet/xTract

\author{
http://bioinformatics.ust.hk/ecl.html \\ http://www.kojak-ms.org \\ https://www.msstudio.ca/crosslinking \\ http://www.biochem.mpg.de/5111795/maxquant \\ http://www.stavrox.com \\ Plug-in zur Crosslink-Analyse \\ Analyse von spaltbaren Crosslinkern
}

http://pfind.ict.ac.cn/index.html

http://prospector.ucsf.edu/prospector/mshome.htm

http://patternlabforproteomics.org/sim-xl/Work.html

http://www.stavrox.com

http://goodlettlab.org/xcomb.php

https://github.com/compomics/xilmass

https://xlinkx2beta.hecklab.com

https://portal.thermo-brims.com

http://prottools.ethz.ch/orinner/public/htdocs/xquest http://proteomics.ethz.ch/cgi-bin/xtract_cgi/index.cgi
Identifizierung, Quantifizierung und Darstellung von Crosslinks

Analyse von spaltbaren Crosslinkern (Node für Thermo Proteome Discoverer 2.2)

Identifizierung und Quantifizierung von Crosslinks. optimiert für Verwendung von isotopenmarkierten Crosslinkern

\section{Software zur Darstellung von Crosslinks}

\begin{tabular}{|c|c|c|}
\hline CX-Circos & http://www.cx-circos.net/user & 2D-Darstellung von Crosslinks mit Chord-Diagrammen \\
\hline DynamXL & http://dynamxl.chem.ox.ac.uk & $\begin{array}{l}\text { 3D-Darstellung und Analyse von Crosslinks, Berücksichtigung } \\
\text { der Proteindynamik }\end{array}$ \\
\hline Jwalk & http://topf-group.ismb.lon.ac.uk/Jwalk/Jwalk-master.zip & Oberflächenabstände zwischen quervernetzten Aminosäuren \\
\hline ProXL & https://github.com/yeastrc/proxl-web-app & $\begin{array}{l}\text { Datenbank zur Analyse und zur 2D/3D-Darstellung von } \\
\text { Crosslink-Datensätzen }\end{array}$ \\
\hline xiNET & http://crosslinkviewer.org & 2D-Darstellung von Crosslinks in Netzwerken \\
\hline Xlink Analyzer & $\begin{array}{l}\text { http://www.beck.embl.de/XlinkAnalyzer.html } \\
\text { https://www.cgl.ucsf.edu/chimera/plugins/plugins.html }\end{array}$ & $\begin{array}{l}\text { 3D-Darstellung von Crosslinks (Plug-in für UCSF Chimera } \\
\text { Programm) }\end{array}$ \\
\hline XLinkDB 2.0 & http://xlinkdb.gs.washington.edu/xlinkdb & $\begin{array}{l}\text { Datenbank und Werkzeuge zum Speichern, Visualisieren und } \\
\text { Vorhersagen von Proteininteraktionen }\end{array}$ \\
\hline
\end{tabular}

Software zur Modellierung von Proteinen und zum Docking innerhalb von Proteinkomplexen

$\begin{array}{lll}\text { HADDOCK } & \text { https://haddock.science.uu.nl } & \text { Proteindocking mit räumlichen Beschränkungen } \\ \text { IMP } & \text { https://integrativemodeling.org } & \text { Proteinmodellieren/Docking mit räumlichen Beschränkungen } \\ \text { I-TASSER } & \text { https://zhanglab.ccmb.med.umich.edu/I-TASSER } & \text { 3D-Proteinstrukturvorhersage mit räumlichen Beschränkungen }\end{array}$

Die quervernetzten Aminosäurepositionen können mit den Kryo-EM-Strukturmodellen abgeglichen werden. Abbildung 2 beschreibt schematisch den Ablauf der Einzelpartikel-Kryo-EM, die zum 3D-Volumenmodell des Komplexes führt. Aus einer großen Anzahl von elektronenmikroskopischen 2D-Bildern des Komplexes werden die unterschiedlichen Orientierungswinkel der Moleküle bestimmt und mit dieser Information im Computer eine dreidimensionale Rekonstruktion der Struktur berechnet. Die Interpretation der berechneten Strukturen in Form von atomaren Modellen erfordert entweder ein bereits vorhandenes Strukturmodell oder eine de novo-Erstellung eines solchen Modells. Eine zuverlässige de novoModellierung der Proteine erfordert jedoch eine Auflösung im Bereich von 3 Å oder besser, die es ermöglicht, nahezu alle Proteinseitenketten in der Kryo-EM-Struktur sichtbar zu machen. Weil letztendlich die meisten der derzeit vorhandenen Kryo-EM-Strukturen eine niedrigere Auflösung besitzen, bieten CX-MS-Daten die Möglichkeit, Proteinbausteine anhand von Cross-Linking-Daten und Homologiemodellen in die Gesamtstruktur einzupassen. Ferner erlaubt CX-MS den Verlauf von intrinsisch ungeordneten Proteinregionen in dem Proteinensemble zu kartieren, selbst wenn diese aufgrund ihrer Dynamik nicht in der 3D-Volumendichte sichtbar sind. Die Strukturmodelle der Proteine werden in das 3D-Volumen des Kryo-EM-Modells eingepasst und die Orientierung der Proteine zueinander mit den CX-MS-Daten abgeglichen.

Das manuelle Einpassen und Modellieren von Proteinen in das 3D-Volumen, basierend auf CX-MS-Daten, ist sehr aufwendig und bedarf einer profunden Kenntnis aller zur Verfügung stehenden biochemischen und genetischen Daten der beteiligten Proteine. Es beschäftigen sich zunehmend Arbeits- gruppen mit der Programmierung von Algorithmen, die solche Prozesse automatisieren (Tab. 1).

\section{Das Spleißosom als Paradebeispiel für CX-MS-Daten in der Strukturanalyse}

Wie CX-MS-Daten zur Anordnung von Proteinen in 3D-Kryo-EM-Strukturen genutzt werden, soll anhand der kürzlich gelösten Strukturen des Spleißosoms [12-16] verdeutlicht werden. Das Spleißosom stellt einen der dynamischsten multifaktoriellen Ribonukleoproteinkomplexe in eukaryotischen Zellen dar. Es katalysiert das Herausschneiden der Introns und die Ligation der Exons einer Prä-mRNA zu einer „reifen“ mRNA und besteht aus fünf kleinen small nuclear-RNAs (snRNAs) und bis zu 170 Proteinen. Das Spleißosom assembliert auf der Prä-mRNA und durchläuft mehrere funktionelle Stufen, die sich in den RNA-RNA-Interaktionen und 
in extrem unterschiedlichen Proteinkompositionen unterscheiden. Diese Dynamik, die flexible Natur der Proteine und das große Molekulargewicht haben die Strukturaufklärung von funktionellen Zuständen des SpleiBosoms lange erschwert. Durch Kryo-EM gelang es in den letzten beiden Jahren, beeindruckende 3D-Strukturen von intakten, funktionellen Spleißosomen zu generieren. Um Proteine in den verschiedenen Zuständen präziser zu lokalisieren und zu positionieren, nutzen wir CX-MS-Daten. Abbildung 3 illustriert ein solches Vorgehen anhand des 3DVolumenmodells des aktivierten Spleißosoms (Bact-Komplex) [13] mit einer Auflösung von 5,8 $\AA$ (Abb. 3A). In das 3D-Volumen wurden die zur Verfügung stehenden Strukturen von einzelnen Proteinen eingepasst. Aufgrund der Auflösung sind ungefaltete Proteinbereiche der spleißosomalen Proteine Prp2, Bud13, Prp45 und Spp2 nicht eindeutig zuzuordnen. Gerade solche Bereiche sind von struktureller und funktioneller Relevanz im Spleißosom, da sie mit multiplen Proteinen sequenziell interagieren können. Diese Bereiche können durch CX-MS in der 3D-Struktur

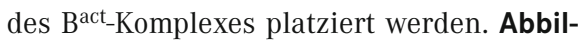
dung 3B zeigt einen vergrößerten Teilbereich ohne 3D-Volumen-Darstellung und nur noch solche Proteine, die definierte Sekundärstrukturelemente aufweisen. Diese Proteine können auch als einfache farbige Flächen mit entsprechend farbigen Aminosäuren, die als Cross-Links zu den ungefalteten Regionen der Proteine Prp2, Bud13, Prp45 und Spp2 identifiziert wurden, dargestellt werden (Abb. 3C, D). Abbildung 3E zeigt dann die entsprechenden Aminosäurereste und schematisch die ungefalteten Proteinregionen der Proteine Prp2, Bud13, Prp45 und Spp2, die dort aufgrund der Cross-Linking-Daten platziert wurden und ein extensives Proteininteraktionsnetzwerk erscheinen lassen. Dieses Netzwerk ist von funktioneller Bedeutung, da vermutlich gerade diese Interaktionen der ungefalteten Proteinregionen das Protein Prp2 „festklemmen“, bevor dieses katalytisch aktiv werden kann und den $B^{\text {act_ }}$ Komplex in einen weiteren funktionellen Zustand, den katalytisch aktivierten B*-Komplex, überführt.

\section{Danksagung}

Die hier gezeigten Arbeiten von R. Lührmann, H. Stark und H. Urlaub werden durch die DFG (SFB860, Integrative Structural Biology of Dynamic Macromolecular Assemblies) unterstützt.

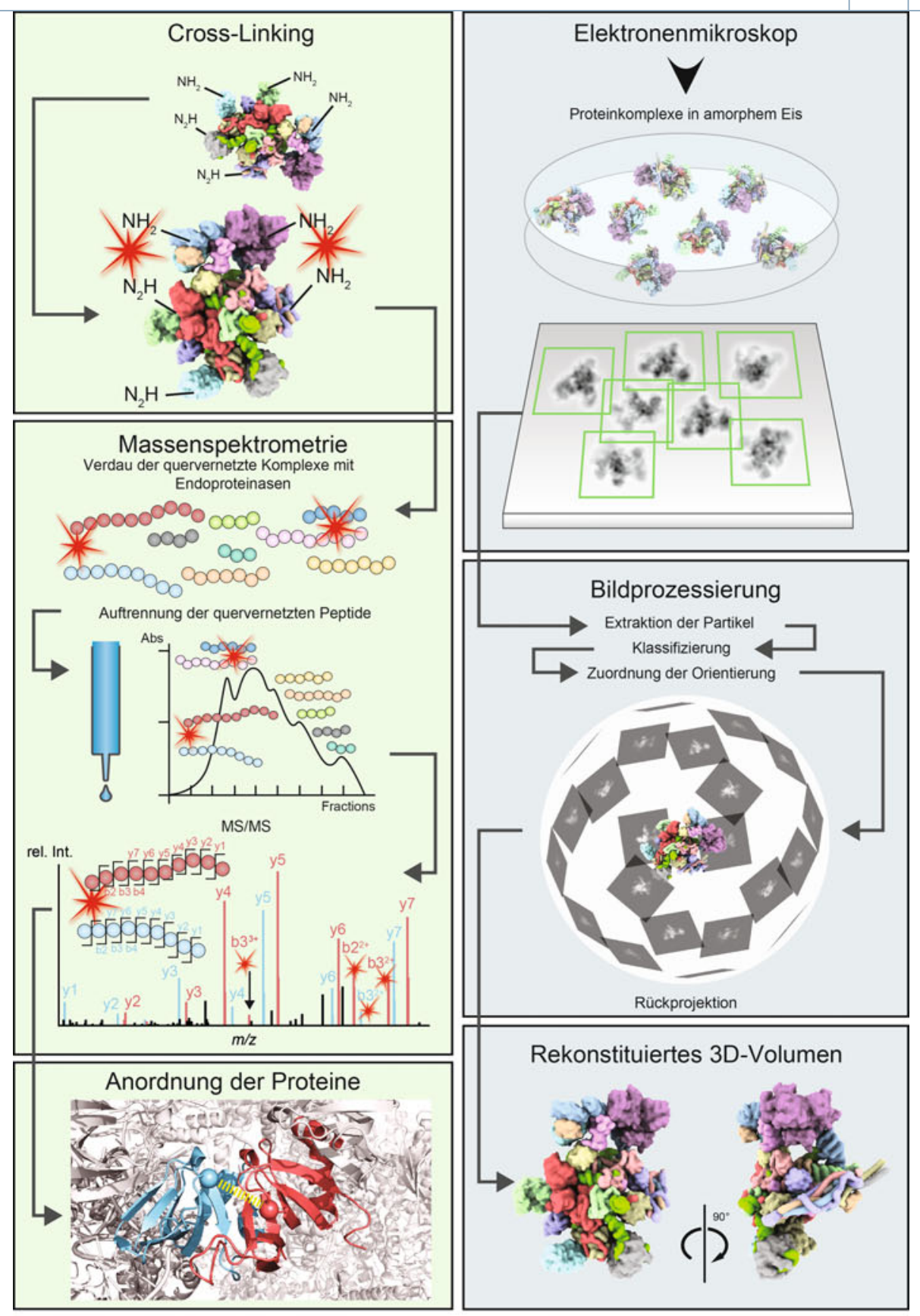

Anordnung aller Proteinstrukturen im 3D-Volumen

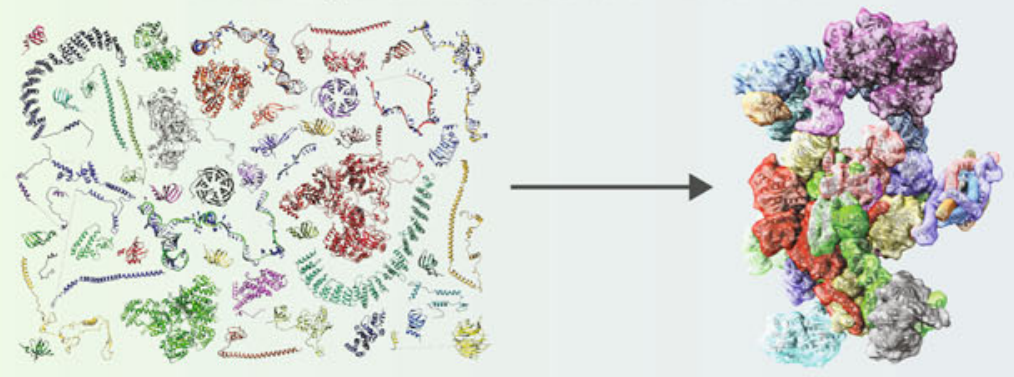

$\Delta$ Abb. 2: Darstellung der CX-MS- und Kryo-Elektronenmikroskopie(EM)-Arbeitsabläufe anhand eines makromolekularen Komplexes. Zur CX-MS-Analyse werden aufgereinigte makromolekulare Proteinkomplexe mit Cross-Linkern inkubiert und anschließend alle Proteine mit Endoproteinasen zu Peptiden verdaut. Quervernetzte Peptide werden mittels Gelfiltration von nicht quervernetzten Peptiden abgetrennt und im Massenspektrometer (MS) sequenziert. Liegen Strukturmodelle der Proteine vor, können die quervernetzten Proteinregionen zueinander angeordnet werden. Zur Kryo-EM-Analyse werden Proteinkomplexe in amorphem Eis eingebettet, und es werden 2D-Projektionen der Komplexe erzeugt, die anschließend nach mehreren Bearbeitungsschritten in dreidimensionale Dichtevolumina rückprojeziert werden. In diesen 3D-Volumina sind die Proteinstrukturen je nach erzielter Auflösung sichtbar und/oder werden mithilfe der CX-MS-Daten und vorliegenden Strukturmodelle der Proteine im 3D-Volumen platziert. 

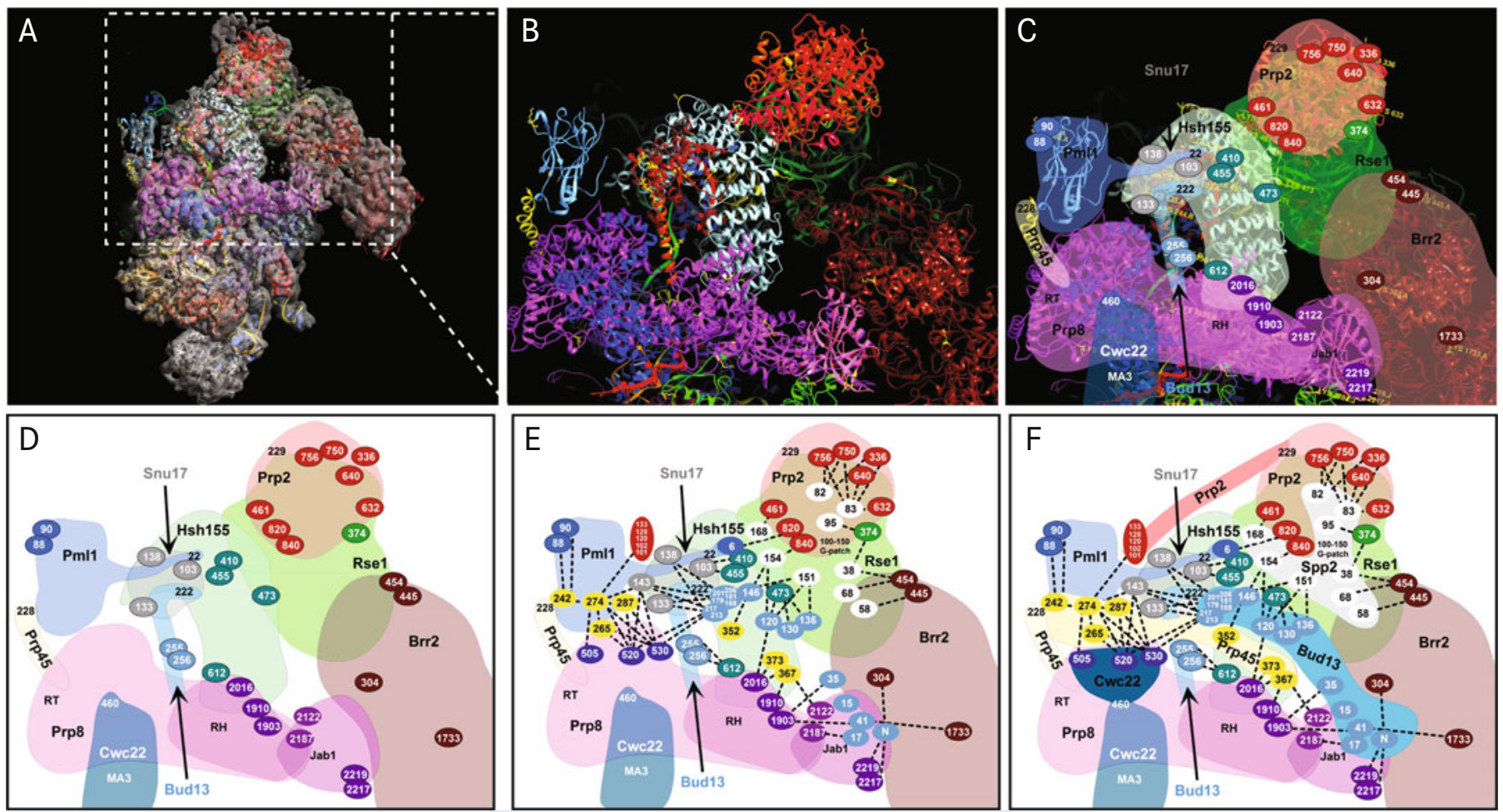

$\triangle$ Abb. 3: Modellierung von ungefalteten Proteinbereichen der spleißosomalen Proteine Prp2, Bud13, Prp45 und Spp2 innerhalb des aktivierten Spleißosoms ( $\mathrm{B}^{\text {act }}$ ) mittels CX-MS-Daten. A, 3D-Struktur des Bact-Spleißosoms bei einer Auflösung von ca. $6 \AA$. Diese Auflösung erlaubt die Platzierung von Strukturen spleißosomaler Proteine mit definierten Proteindomänen. Nicht sichtbar hingegen sind Proteine bzw. Domänen mit großen ungefalteten Bereichen. B, Bereich des Spleißosoms ohne 3D-Volumendichte mit den Strukturen der spleißosomalen Proteine Cwc22, Prp45, Pml1, Hsh 155, Prp2 und Brr2. C, Darstellung der Proteine als farbige Flächen und deren Aminosäuren (Lysine in den entsprechenden Farben der Proteine), die mittels Massenspektrometrie zu anderen Proteinen quervernetzt gefunden wurden. D, wie C, nur ohne Strukturmodelle der entsprechenden Proteine. E, schematische Darstellung der ungefalteten Bereiche der Proteine Cwc22, Prp45, Prp2, Spp2 und Bud 13 mit ihren quervernetzten Aminosäuren zu anderen Proteinen im Komplex. Die identifizierten Cross-Links erlauben die Platzierung der nicht gefalteten Bereiche der Proteine Cwc22, Prp45, Prp2, Spp2 und Bud 13 in diesen Bereich des Spleißosoms.

\section{Literatur}

[1] Cheng Y (2015) Single-particle cryo-EM at crystallographic resolution. Cell 161:450-457

[2] Cheng Y, Grigorieff N, Penczek PA et al. (2015) A primer to single-particle cryo-electron microscopy. Cell 161:438-449 [3] Nogales E, Scheres SH (2015) Cryo-EM: a unique tool for the visualization of macromolecular complexity. Mol Cell 58:677-689

[4] Fernandez-Leiro R, Scheres SH (2016) Unravelling biological macromolecules with cryo-electron microscopy. Nature 537:339-346

[5] Schmidt C, Urlaub H (2017) Combining cryo-electron microscopy (cryo-EM) and cross-linking mass spectrometry (CX-MS) for structural elucidation of large protein assemblies. Curr Opin Struct Biol 46:157-168

[6] Sinz A (2003) Chemical cross-linking and mass spectrometry for mapping three-dimensional structures of proteins and protein complexes. J Mass Spectrom 38:1225-1237

[7] Rappsilber J (2011) The beginning of a beautiful friendship: cross-linking/mass spectrometry and modelling of proteins and multi-protein complexes. J Struct Biol 173:530-540 [8] Leitner A, Faini M, Stengel F et al. (2016) Crosslinking and mass spectrometry: an integrated technology to understand the structure and function of molecular machines. Trends Biochem Sci 41:20-32

[9] Kao A, Chiu CL, Vellucci D et al. (2011) Development of a novel cross-linking strategy for fast and accurate identification of cross-linked peptides of protein complexes. Mol Cell Proteomics 10, doi: 10.1074/mcp.M110.002212

[10] Muller MQ, Dreiocker F, Ihling CH et al. (2010) Cleavable cross-linker for protein structure analysis: reliable identification of cross-linking products by tandem MS. Anal Chem 82:6958-6968

[11] Liu F, Rijkers DT, Post H et al. (2015) Proteome-wide profiling of protein assemblies by cross-linking mass spectrometry. Nat Methods 12:1179-1184
[12] Agafonov DE, Kastner B, Dybkov O et al. (2016) Molecular architecture of the human U4/U6.U5 tri-snRNP. Science 351:1416-1420

[13] Rauhut R, Fabrizio P, Dybkov O et al. (2016) Molecular architecture of the Saccharomyces cerevisiae activated spliceosome. Science 353:1399-1405

[14] Bertram K, Agafonov DE, Dybkov O et al. (2017) Cryo-EM structure of a pre-catalytic human spliceosome primed for activation. Cell 170:701-713

[15] Bertram K, Agafonov DE, Liu WT et al. (2017) Cryo-EM structure of a human spliceosome activated for step 2 of splicing. Nature 542:318-323

[16] Haselbach D, Komarov I, Agafonov DE et al. (2018) Structure and conformational dynamics of the human spliceosomal Bact complex. Cell 172:454-464

Open Access:

This article is distributed under the terms of the Creative Commons Attribution 4.0 International License (http://creativecommons.org/licenses/by/4.0/), which permits use, duplication, adaption, distribution and reproduction in any author(s) and the source, provide a link to the Creative Commons license, and indicate if changes were made.

pen access funding provided by Max Planck Society.

\section{Korrespondenzadresse:}

Prof. Dr. Henning Urlaub

Bioanalytische Massenspektrometrie

Max-Planck-Institut für biophysikalische Chemie Am Faßberg 11

D-37077 Göttingen

Tel.: 0551-201-1060

Fax: 0551-201-1197

hurlaub@gwdg.de

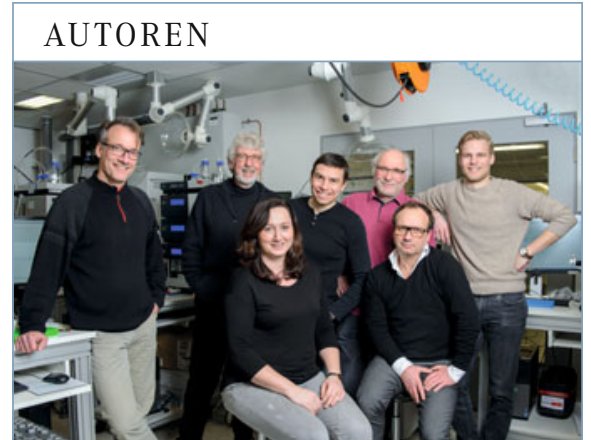

Holger Stark, Reinhard Lührmann, Alexandra Stützer, Olexandr Dybkov, Berthold Kastner, Henning Urlaub und Karl Bertram (v. I.n. r.)

Die Forschungsgruppe „Bioanalytische Massenspektrometrie“ am Max-Planck-Institut für biophysikalische Chemie arbeitet an der Analyse von Proteinen, Proteinkomplexen, Interaktionen zwischen Proteinen und Nukleinsäuren mithilfe von hochmodernen Massenspektrometern. Die Proben werden hauptsächlich mit Elektrosprayionisierung- und in speziellen Anwendungen mit Matrix-unterstützte Laser-Desorption/Ionisation(MALDI)-Massenspektrometrie analysiert. 